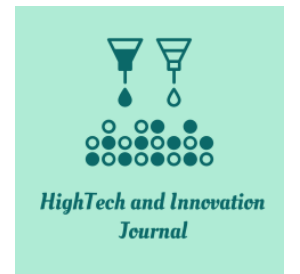

ISSN: 2723-9535

\title{
AHP Approach for Determining Category in Social Media Content Creation in Order to Maximize Revenue per Mille (RPM)
}

\author{
Kevin Joseph De Guzman ${ }^{1 *}$, Rex Aurelius C. Robielos ${ }^{1}$ \\ ${ }^{I}$ School of Industrial Engineering and Engineering Management, Mapua University, Intramuros, Manila, Philippines.
}

Received 20 November 2021; Revised 22 January 2022; Accepted 24 January 2022; Published 01 March 2022

\begin{abstract}
This study utilizes the Analytic Hierarchy Process (AHP) in the selection of an optimal niche or category of videos for maximizing view count. The main income from videos is derived from RPM, which is a set amount per thousand views. A set of criteria was determined from attributes in the dataset that logically contribute to either the videos' SEO or trend/popularity. The criteria in question were also determined by commonalities across a vast number of video content platforms, which focus more on the essential attributes of a video. In order to perform pairwise comparison, weights were derived from coefficients generated using Linear Regression. Following the creation of the model, we identify the categories with the highest potential for gaining views. Based on the results, the study may be performed in another time frame to reflect the major shifts in public interest over time. Thus, the importance of its repeatability and degree of usability across datasets from different platforms is emphasized.
\end{abstract}

Keywords: Analytical Hierarchy Process; Regression Analysis; Video Content Creation; Social Media.

\section{Introduction}

In contemporary society, social media is changing the way people create, share, and consume information [1]. These social media platforms are being driven by the content created by and for their users. These types of content are known as User Generated Content (UGC). Being a content producer on these platforms is becoming a more viable way to earn an income in the creative space. The content production through social media allow users to fulfil their information, entertainment, and mood management needs, while its generation (or sharing) allows for self-expression and self-actualization [2]. The exponential growth of social media in contemporary society makes them necessary tools for communication, content creation, sharing, and business growth [3].

With more and more creators present on a platform, competition can be detrimental to success since viewership share is diluted by more participants. One other factor is the introduction of content from traditional media companies, usually in the form of video clips of shows broadcast on television networks and cable networks. These types of content are called non-user generated content. For traditional media companies interested in entering the internet media market, one would have to use one of the many video sharing platforms. They have the capacity and capability to produce content on any topic or category. However, not all content posted on video platforms such as YouTube gets the desired attention, and only a fraction can reach a large audience, particularly the videos posted by social media marketers expecting millions of views [4-8]. Companies want to identify these categories with the highest potential for a high number of views on the platform, thereby maximizing profit while considering parameters affecting the quality and timeliness of video production and publishing.

* Corresponding author: kevindeguzman129@gmail.com

$>$ This is an open access article under the CC-BY license (https://creativecommons.org/licenses/by/4.0/).

(C) Authors retain all copyrights. 
In this paper, we measure the characteristics of videos from different categories, in terms of video duration, view counts, and user engagement, and assess their potential for revenue. By understanding the characteristics of videos with high view counts, the study will help traditional media companies determine the type of content they would want to produce to ensure returns on investment. Based on our initial observations of the data [9], we discovered that the videos in different categories have noticeably different characteristics. This study aims to identify categories with the potential for high view counts, using criteria common to most video sharing platforms. The study also aims to formulate a methodology that can be easily reused against different video sharing platforms, as well as across time periods.

\section{Methods}

\subsection{Dataset}

The data is sourced from video sharing websites from 2017 (N 200,000). The features we consider for each video are video length (in seconds), number of views, category names, video resolution, the word count of the title, and the word count of the tags used. Features common to social media platforms are functions to boost social interaction [10], such as, the user's ability to post comments on a video, like or dislike a video, or share a video on other social networking platforms such as Facebook or Twitter. For this study, we will incorporate likes and dislikes and/or their ratio.

The features that were identified for this study can be found on most, if not all, social media platforms, especially for video content. This is to enable the methodology to be used easily across datasets from the different platforms, which in and of themselves have different priorities. For example, some platforms only permit short form videos, while platforms directed at gaming content generally have hour-long videos. Another example is with categories, where there are different methods of categorization per platform.

\subsection{Linear Regression in Calculating Criterion Weights}

In the Analytic Hierarchy Process (AHP), the decision maker makes comparisons between pairs of attributes or alternatives. In real applications the comparisons are subject to judgmental errors. Based on this model we present the formulae for the evaluation of the estimates of the AHP-weights obtained by regression analysis [11].

For the calculation of weight for each criterion, we utilized Linear Regression to estimate weights from the resulting coefficients. The target variable would be view count as it is the primary driver of assessing rate of income on any video sharing platform. The model is presented in statistical formula as follows:

$\widehat{V}=b_{o}+b_{1} X_{1}+b_{2} X_{2}+b_{3} X_{3}+b_{4} X_{4}+b_{5} X_{5}$

Where:

$\widehat{V}$ : Views;

$X_{1}$ : Length;

$X_{2}$ : Quality;

$X_{3}$ : Title Length Count;

$X_{4}$ : Tag Word Count;

$X_{5}$ : Like to Dislike Ratio, denoted as Rating.

\subsection{Linear Regression in Calculating Alternative Weights with regards to each Criterion}

Using the same process as with assessing weights for criteria, the researcher performed the same with regards to each category. As with any topics that can be viewed on the platform, not all of them can be produced in such a way that exemplify the attributes identified as primary success factors. In order to perform regression on multiple categorical variables, the categories are coded into its' own matrix of Boolean values. A sample of the model is shown below:

$\widehat{C_{1}}=b_{o}+b_{1} X_{1}+b_{2} X_{2}+b_{3} X_{3}+b_{4} X_{4}+\cdots+b_{n} X_{n}$

Where:

$\widehat{C_{1}}:$ Criterion Coefficient

$X_{n}$ : Category n (as parsed and identified from the dataset)

This process is repeated for each criterion. With this we can proceed with presenting the AHP model with the weights being provided by the above methods. Then, pairwise comparisons are made and calculated after estimation of 
weights from applying linear regression against criteria and against the category with regards to the criteria. This was done via Python using Numpy, Scikit-Learn, Pandas, as well as other utility functions.

\subsection{Analytic Hierarchy Process (AHP) Model}

Figure 1 shows the 4 layers of AHP. The first layer shows the main goal, which is the identification of the category/topic to focus on for maximum potential views. The primary criteria the researched has selected are production quality, search engine optimization, and user engagement. The secondary criteria that the researcher has selected are length, quality, \# of words in the video title, \# of tags used to describe the video, or by votes, ratings, likes or dislikes.

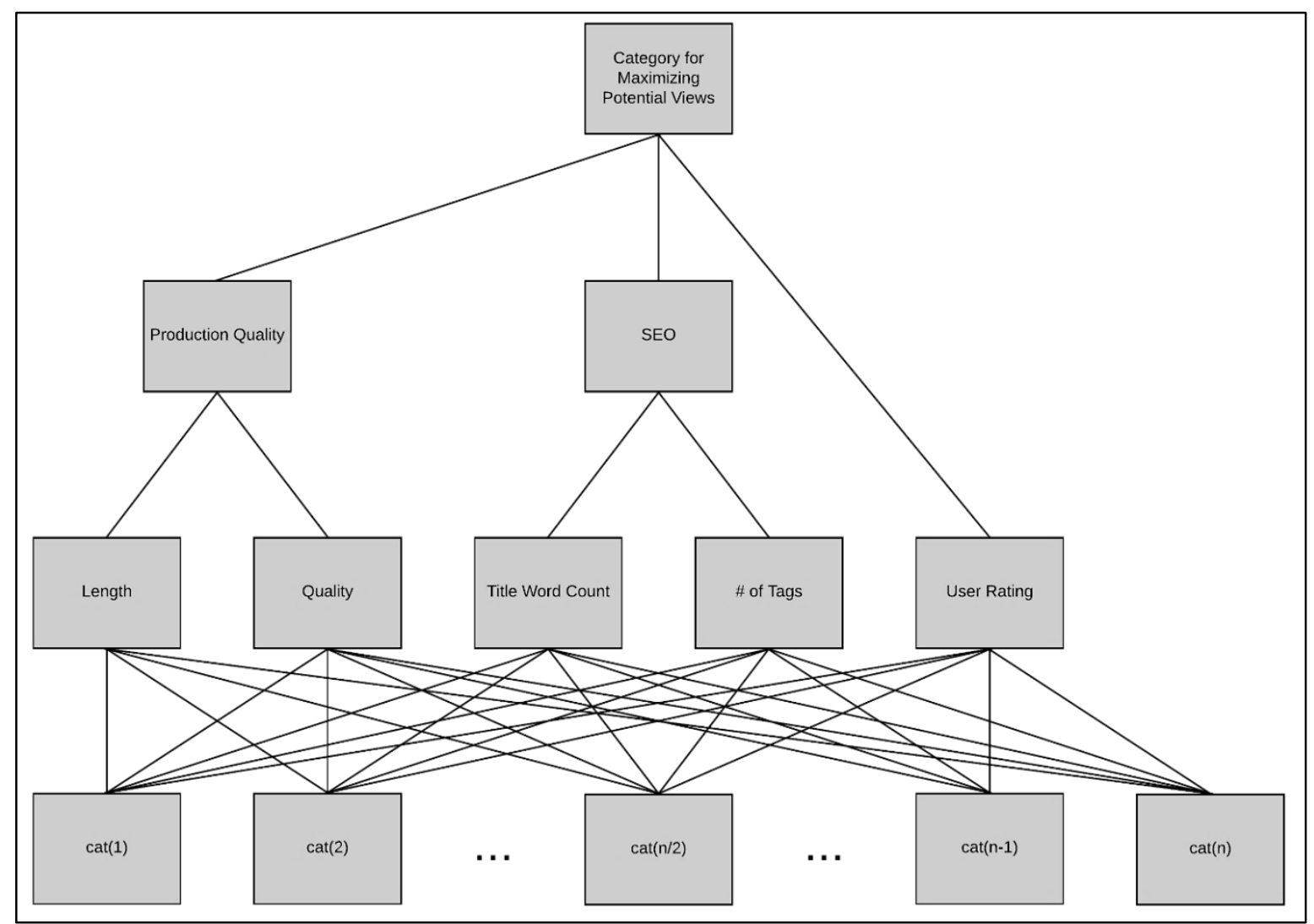

Figure 1. Generalized AHP Model

These criteria are generally the parameters content creators tune during the production process. Length is an important decision to make as it is highly correlated with production time. Content producers must decide on a balance of production time and quality to ensure a consistent upload schedule. Video quality is another primary factor as this pertains to the actual recording quality, as well as production value. Tags improve the videos' visibility in the platform's search functionality. User rating reflects how well the videos are received by viewers, this may depend on the presentation (positive/negative, conservative/controversial) of a specific issue or topic within the general category.

Also, videos with higher ratio of likes per view, higher negative sentiment in comments, and higher view count are more likely to be watched [12]. Higher user engagement in any kind of way is more likely to be shared, in either to continue the discussion or to have their social network have a say in the content of the video.

Videos from different categories have different statistics on video duration, popularity, user engagement and so on [9], and thus each category have different priorities in which to maximize views. Video platforms permits users to share different categories of videos with different groups of people [13]. Thus, we believe that different types or categories of video (e.g., music, comedy, drama, and animation) may affect view count differently.

\section{Results}

\subsection{Dataset Exploration and Pre-Requisites for Linear Regression}

From the dataset, alternatives were generated from the unique categories of the data used, which resulted into 84 categories. Categorical data is converted into binary columns before performing linear regression. Data was processed in Python using standard statistical libraries. 
For the features in the dataset, shown from a sample set in Figure 2, we have video length in seconds, number of views, number of likes and dislikes, the number of tags, and the word count of the title. We observe that there can be cases where two (or more) categories can be tied to a video. This will be considered upon creation of the model through the means of coding. Aside from this we also have the quality variable denoted as "hd" in the dataset. Further inspection of the likes and dislikes, we can simplify into a singular value as a ratio between the two. This is to remove multi-collinearity between likes and dislikes.

\begin{tabular}{|c|c|c|c|c|c|c|c|c|}
\hline length & nb_views & cate & gories & hd & title_word_count & tag_count & likes & dislikes \\
\hline 1583.0 & $12 \overline{7} 450.0$ & & cat14 & False & $\begin{array}{lll}- & - & 5\end{array}$ & 3 & 4282.0 & 816.0 \\
\hline 2501.0 & 480620.0 & & cat78 & False & 15 & 6 & 15188.0 & 4037.0 \\
\hline 1513.0 & 99720.0 & & cat18 & False & 6 & 9 & 3071.0 & 917.0 \\
\hline 1710.0 & 598820.0 & cat13 & cat53 & False & 13 & 3 & 19162.0 & 4791.0 \\
\hline 1694.0 & 155850.0 & & cat 60 & False & 13 & 8 & 4925.0 & 1309.0 \\
\hline
\end{tabular}

Figure 2. Sample of the Dataset

Looking at the summary statistics of the dataset in Figure 3, we see that the average video length is about 15 minutes, with the minimum of 5 seconds. This may be due to having videos from short duration video platforms line Vine or TikTok, re-published to other video sharing websites. Videos longer that 15 minutes tend to be educational in nature, or in the form of radio shows and/or podcasts on a myriad of topics. An appealing study on user generated content illustrated the result of difference video popularity and length between user generated content and non-user generated content [14-16]. A mean view count of around 420k shows us that most of the videos on the dataset are somewhat popular in nature. Title and word count seem to be in close correlation, as tags can be derived from keywords used in video titles. Some such practices are called keyword brainstorming in the SEO space.

\begin{tabular}{|c|c|c|c|c|c|c|}
\hline & length & nb_views & title_word_count & tag_count & likes & dislikes \\
\hline count & 191179 & 191179 & $\overline{191179}$ & 191179 & 191179 & 191179 \\
\hline mean & 839.566 & 417419 & 12.7108 & 6.46612 & 13173.7 & 3523.03 \\
\hline std & 2895.35 & $1.03745 e+06$ & 3.39349 & 2.70153 & 32177.4 & 10029.3 \\
\hline $\min$ & 5 & 1020 & 1 & 1 & 31 & 10 \\
\hline $25 \%$ & 375 & 85270 & 11 & 5 & 2586 & 758 \\
\hline $50 \%$ & 601 & 172350 & 13 & 6 & 5366 & 1473 \\
\hline $75 \%$ & 1065 & 381750 & 15 & 8 & 12071.5 & 3160 \\
\hline $\max$ & $1.21852 \mathrm{e}+06$ & $1.1346 \mathrm{e}+08$ & 19 & 19 & $3.04073 e+06$ & $1.49767 e+06$ \\
\hline
\end{tabular}

Figure 3. Summary Statistics of Numerical Data in Dataset

\subsection{Pre-Requisites for Linear Regression}

One of the prerequisites in utilizing linear regression is to verify if the independent variables follow a normal distribution. A normal distribution is a probability distribution of outcomes that is symmetrical or forms a bell curve. In a normal distribution $68 \%$ of the results fall within one standard deviation and $95 \%$ fall within two standard deviations. In order to visualize this, we need to plot the logarithmic values of the independent variables individually.

Inspecting the log density of the numerical variables in Figure 4, we could see that almost all, apart from tag count, can be considered a normal distribution. This shows that most variables are appropriate to be used in linear regression in order to get weights from the coefficients.

\subsection{Linear Regression}

From the results, we see that that the R-score for the linear regression model to predict view count from the independent variables (length, quality, title/tag word count, and like to dislike ratio), would be around 0.02 , which is quite low. Regardless of the R-squared, the significant coefficients still represent the mean change in the response for one unit of change in the predictor while holding other predictors in the model constant. As such, we can still draw important conclusions about how changes in the predictor values are associated with changes in the response value.

For the weighs of alternatives shown in Table 1, we can see that quality has the highest $\mathrm{R}$ score from the criteria, followed by tag word count, rating, title word count, with length having the smallest score. With average network throughput increasing year-by-year, it is apparent that more and more users demand content produced in higher fidelity. Improvements in screen resolution for both mobile devices and home systems affect viewing experience negatively when consuming lower quality videos. 


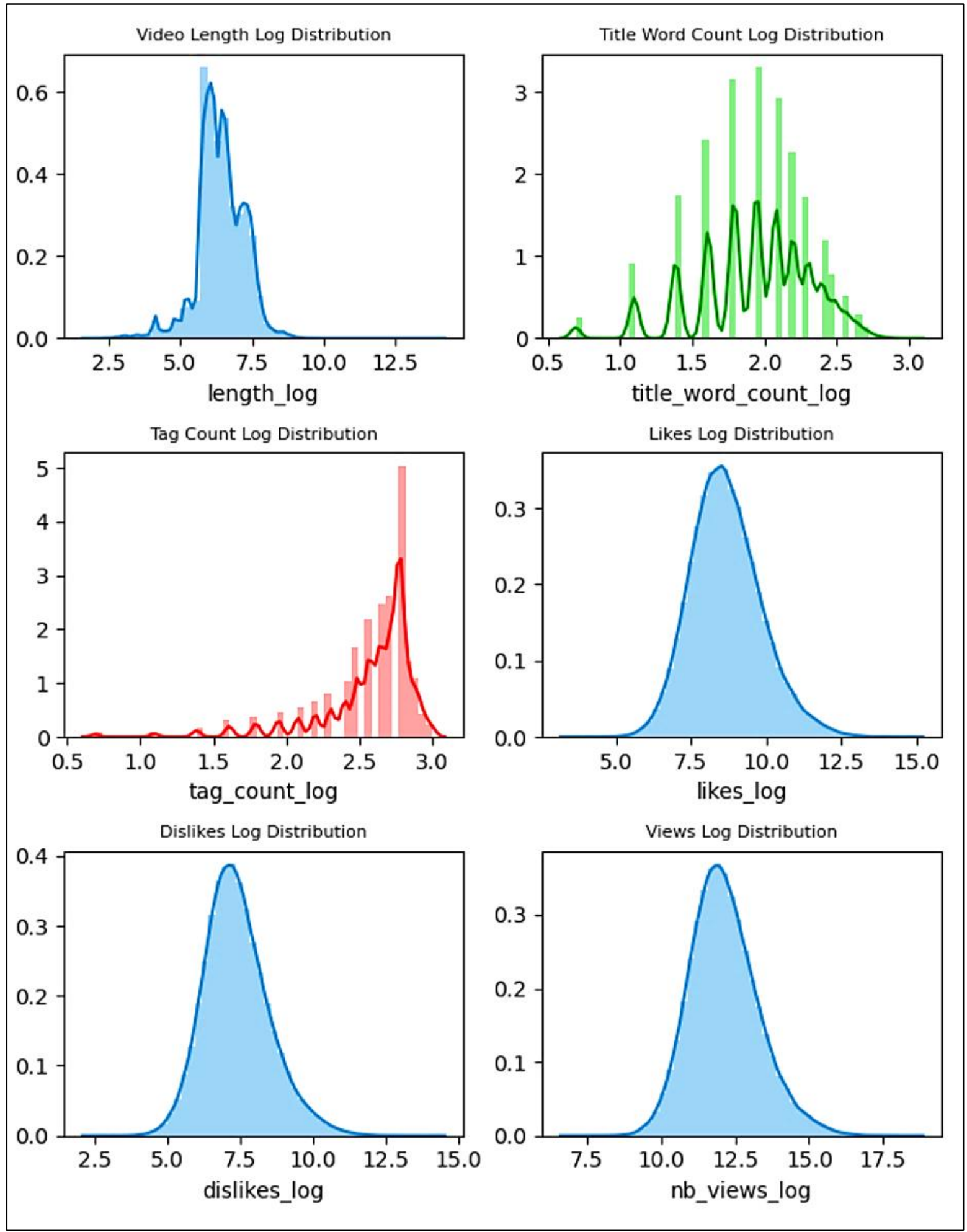

Figure 4. Log Distribution of Primary Independent Variables

Table 1. Identified Criteria with Calculated Weights

\begin{tabular}{ccc}
\hline Criteria & Coefficient & Weight \\
\hline Length & 3.575928 & 0.000032 \\
Quality & 57838.21097 & 0.510905 \\
Title Word Count & 11411.26086 & 0.1008 \\
Tag Word Count & 28163.24316 & 0.248776 \\
Rating & 15791.0216 & 0.139488 \\
\hline
\end{tabular}


Table 2 presents the results after the creation of the linear regression models to verify how well the metrics affect the primary criteria. It is apparent that video quality holds the most weight in determining the popularity of a video. Tag count also has a significant effect on views as it positively affects the videos' visibility in search results as well as their being correctly identified in profiling algorithms. It will be more likely to be suggested to viewers who have also watched videos that have similar tags.

Table 2. Table of $\mathbf{R}$ Scores for model with regards to each criterion

\begin{tabular}{cc}
\hline Criteria & R Score \\
\hline Length & 0.006244838 \\
Quality & 0.990978027 \\
Title Word Count & 0.074178505 \\
Tag Word Count & 0.273546934 \\
Rating & 0.139005149 \\
\hline
\end{tabular}

User rating follows tag count in the comparison of R scores. Videos suggested based on the profiling of viewers' interests will also have a higher likelihood of being liked, commented on, and shared by those users. Viewers who are trying to find content outside their usual interests may be attracted to a video with a higher level of user engagement. Title word count has a slightly less significant score than that of tag count. It may be due to the limitations of how much information one is able to include in such a small space. At most times, titles are used to entice viewers to misleading to the actual content, aptly named "click bait" titles.

Using more tags that describe the video in order to increase visibility in search engines also proves to be a good indicator that it will have more views. Keywords taken from either the video's title or determined through keyword brainstorming can be an effective way of capturing more viewer share. Scores from user engagement, either through voting or a like/dislike system, also prove to be a good metric in determining whether a specific video gets more views. As more and more active viewers engage with the content through the provided means, it is more likely to be shared across their own social networks, thus increasing the view count even further. Title word count has a significantly lower weight when compared to tag count, as tags don't have the limitation of character or word length. Some context may be lost when titles must be able to convey the essence of the video in such a short space. In certain videos, titles and tags are used in complement, with having a catchy title not exactly describing what the video entails, while having tags correctly identify content for target viewers.

The lowest weight was video length. As seen from the summary statistics, videos come in many lengths and forms, from 5 seconds to a couple of hours. As far as the results show, video length does not entirely matter in terms of potential view counts. Shorter videos can be consumed easily, while longer videos tend to have more potential for revenue outside of view counts, such as advertisements that are part of the production, or advertisements inserted by the platform in the middle of videos. With these primary weights, we can proceed to calculate the weights for the alternatives for each of the criteria.

\subsection{AHP Weight Calculation}

Table 3 shows the calculated weights for the top 5 categories, with weights against each criterion, with the final column being the final composite weight. As the previous section observed the weights regarding views, this section will discuss observations made on the distribution of the weights for each category. Table 3 shows that the top two categories were concerned with video/production quality. However, the 2nd top category's composite weight (cat42) holds close to the top category even though it has a significantly lower weight on the length criteria. This may prove to show that video length does not entirely matter if the video production quality is high. The 3rd category (cat5) might describe average-length videos but with lower video quality. Its composite weight is being raised by higher quality or number of tags, as well as its user rating, which has the highest weight of the top 3 categories.

Table 3. Top Categories from the Utilized Methodology

\begin{tabular}{cccccccc}
\hline Alternative & Weight vs. Length & Weight vs. Quality & Title Word Count & Tag Word Count & Weight vs. Rating & Composite Weight \\
\hline cat34 & 0.03421495 & 0.4067542 & 0.041242 & 0.023159 & 0.002266 \\
cat42 & 0.0048955 & 0.40560906 & 0.021549 & 0.010997 & 0.011571 \\
cat5 & 0.01885897 & 0.08716162 & 0.013686 & 0.014179 & 0.014565 \\
cat29 & 0.00130151 & 0.01740777 & 0.036413 & 0.009682 & 0.051471 \\
cat71 & 0.00818821 & 0.00266157 & 0.013987 & 0.012763 & 0.015335 & 0.013788 \\
\hline
\end{tabular}




\section{Conclusion}

The utilization of linear regression for estimating weights in AHP proved to be a good approach for processing data generated from high-traffic social media platforms. It can remove biases that can come from small sample sizes, such as surveys directed at a handful of executives. The more data-driven research becomes, it is inherently more reliable, and the more easily it can be implemented in other industries or subject matters. The independent variables in the study were chosen with the importance of being able to apply the methodology across different video platforms, as well as being easy to repeat in periodic time frames. This is to easily capture shifts in trends, changes in categorization, as well as changes in how videos are measured.

From this study, we have found that for the dataset used, video length does not matter as much as view count. Also, we have found out that while some attributes have consistent weights across most categories, like search engine optimization related attributes (titles, tags), some categories value other attributes more than those of other categories. This can be useful to media companies, or other individuals who peruse the methods in this study, to selectively control how a production should be made for the categories they have selected. It can also be noted that the study did not select a singular category. Instead, the study presents the top categories by their composite weight. This is to further express that different categories have characteristics that may be better or worse than those of their peers, with composite weights being relatively equal. This would mean that the individuals who may use this model can have more control over the final decision of selecting a category according to their priority over certain video attributes.

\section{Declarations}

\subsection{Author Contributions}

Conceptualization, K.J.D.; methodology, K.J.D.; software, K.J.D.; validation, K.J.D.; formal analysis, K.J.D.; investigation, K.J.D.; resources, K.J.D.; data curation, K.J.D.; writing — original draft preparation, K.J.D.; writingreview and editing, K.J.D. and R.R.; visualization, K.J.D.; supervision, R.R.; project administration, K.J.D.; funding acquisition, K.J.D. All authors have read and agreed to the published version of the manuscript.

\subsection{Data Availability Statement}

The data presented in this study are available in article.

\subsection{Funding}

The authors received no financial support for the research, authorship, and/or publication of this article.

\subsection{Declaration of Competing Interest}

The authors declare that they have no known competing financial interests or personal relationships that could have appeared to influence the work reported in this paper.

\section{References}

[1] Mangold, W. G., \& Faulds, D. J. (2009). Social media: The new hybrid element of the promotion mix. Business Horizons, 52(4), 357-365. doi:10.1016/j.bushor.2009.03.002.

[2] Shao, G. (2009). Understanding the appeal of user-generated media: a uses and gratification perspective. Internet Research, 19(1), 7-25. doi:10.1108/10662240910927795.

[3] Kaplan, A. M., \& Haenlein, M. (2010). Users of the world, unite! The challenges and opportunities of Social Media. Business Horizons, 53(1), 59-68. doi:10.1016/j.bushor.2009.09.003.

[4] Khan, G. F., \& Vong, S. (2014). Virality over youtube: An empirical analysis. Internet Research, 24(5), 629-647. doi:10.1108/IntR-05-2013-0085.

[5] Helming, A. G., Adler, D. S., Keltner, C., Igelman, A. D., \& Woodworth, G. E. (2021). The Content Quality of YouTube Videos for Professional Medical Education: A Systematic Review. Academic Medicine, 96(10), 1484-1493. doi:10.1097/ACM.0000000000004121.

[6] Buntain, C., Bonneau, R., Nagler, J., \& Tucker, J. A. (2021). YouTube Recommendations and Effects on Sharing across Online Social Platforms. Proceedings of the ACM on Human-Computer Interaction, 5(CSCW1), 1-26. doi:10.1145/3449085.

[7] Budzinski, O., Gaenssle, S., \& Lindstädt, N. (2020). The Battle of YouTube, TV and Netflix - An Empirical Analysis of Competition in Audio-visual Media Markets. SSRN Electronic Journal, 1(9), 1-26. doi:10.2139/ssrn.3569553.

[8] Sashittal, H. C., \& Jassawalla, A. R. (2021). Brands as personal narratives: learning from user-YouTube-brand interactions. Journal of Brand Management, 28(6), 657-670. doi:10.1057/s41262-021-00248-4. 
[9] Yang, W., \& Qian, Z. (2011). Understanding the characteristics of category-specific YouTube videos. In Entstanden im Rahmen eines Informatikseminars an der kanadischen Simon Fraser University, Burnaby, Canada.

[10] Benevenuto, F., Duarte, F., Rodrigues, T., Almeida, V., Almeida, J., \& Ross, K. (2008). Understanding video interactions in youtube. MM'08 - Proceedings of the 2008 ACM International Conference on Multimedia, with Co-Located Symposium and Workshops, 761-764. doi:10.1145/1459359.1459480.

[11] Laininen, P., \& Hämäläinen, R. Analyzing AHP-matrices by Regression. European Journal of Operational Research, 148, 514-5241010160377-221702 00430-7.

[12] Park, M., Naaman, M., \& Berger, J. (2016). A data-driven study of view duration on YouTube. Proceedings of the 10th International Conference on Web and Social Media, ICWSM 2016, Cologne, Germany, 651-654.

[13] Lange, P. G. (2007). Publicly private and privately public: Social networking on YouTube. Journal of Computer-Mediated Communication, 13(1), 361-380. doi:10.1111/j.1083-6101.2007.00400.x.

[14] Cha, M., Kwak, H., Rodriguez, P., Ahnt, Y. Y., \& Moon, S. (2007). I tube, you tube, and everybody tubes: Analyzing the world's largest user generated content video system. Proceedings of the ACM SIGCOMM Internet Measurement Conference, IMC, 1-14. doi:10.1145/1298306.1298309.

[15] Tu, Z., Wang, Y., Birkbeck, N., Adsumilli, B., \& Bovik, A. C. (2021). UGC-VQA: Benchmarking Blind Video Quality Assessment for User Generated Content. IEEE Transactions on Image Processing, 30, $4449-4464$. doi:10.1109/TIP.2021.3072221.

[16] Wei, J., Yang, X., \& Dong, Y. (2021). User-generated video emotion recognition based on key frames. Multimedia Tools and Applications, 80(9), 14343-14361. doi:10.1007/s11042-020-10203-1. 